\title{
Anmerkungen zur Konkurrenz von Präventionsstaat und Sozialstaat
}

\section{Einleitung}

Die vergangenen Jahre waren innenpolitisch geprägt von zwei scheinbar voneinander unabhängigen Entwicklungen, dem Abbau des Sozialstaates einerseits und dem Ausbau des insbesondere von seinen Kritikern so genannten Präventionsstaates andererseits. Der deutsche Sozialstaat ist, darüber scheint im Wesentlichen Einvernehmen zu bestehen, auf Grund demographischer Entwicklungen und einer von der Globalisierung geprägten Wirtschaftslage an die Grenzen seiner Kapazität gestoßen und bedarf einer grundlegenden und gerade in Wahlkampfzeiten heftig diskutierten Reform seiner Sicherungssysteme. Kaum weniger intensiv wird nicht erst, aber vor allem seit den Terroranschlägen des 11.09.2001 die Frage erörtert, ob sich unser Staat in Sachen innerer Sicherheit zu einem Präventionsstaat entwickelt oder bereits entwickelt hat. Im Folgenden wird der Versuch unternommen, beide Phänomene in einen gemeinsamen Kontext einzufügen: in den der paradigmatischen Ablösung des Sozialstaats durch den Präventionsstaat, also des Perspektivwechsels von der sozialen zur inneren Sicherheit. ${ }^{1}$

\section{Paradigmenwechsel in den Staatszwecken $?^{2}$}

Die Empirie dürfte zwanglos bestätigen, dass ungeachtet aller anderen, auch essentielleren verfassungsrechtlichen Gründe für die Existenz eines Staates unsere staatliche Verfasstheit von der Bevölkerung selbst wenigstens zur Zeit noch maßgeblich als Sozialstaat wahrgenommen werden dürfte. ${ }^{3}$ Die in den Jahrzehnten nach dem zweiten Weltkrieg in einem bis dahin unbekannten Ausmaß ausgebauten sozialen Leistungen sind, diese Behauptung lässt sich schon mit einem Verweis auf den Anteil des Etats der einschlägigen Ministerien am staatlichen Gesamthaushalt ${ }^{4}$ belegen, zweifelsohne

1 Die soziale Gerechtigkeit (dazu zuletzt Eichenhofer, JZ 2005, 209 ff. und, allerdings unscharf trennend, Prantl, Kein schöner Land, 2005), neben der sozialen Sicherheit zweites großes Anliegen des Sozialstaats (vgl. Stern, Staatsrecht I, 2. Auflage 1984, § 21 I 5c, II) ist von dieser Entwicklung weniger evident betroffen. Das mag neben zahlreichen anderen Gründen auch daran liegen, dass der Staat, man denke nur an das derzeit diskutierte Antidiskriminierungsgesetz (dazu Armbrüster, ZRP 2005, 41 ff.), hier auch in der Lage ist, sich monetär zumindest unmittelbar neutraler Maßnahmen zu bedienen.

2 Zum Begriff der Staatszwecke siehe Isensee, in: Isensee/Kirchhof (Hrsg.), HdbStR III, 2. Auflage 1996, § 57 Rn 115 ff.. Genau genommen müsste im vorliegenden Fall zwischen dem Staatszweck Sicherheit und dem Staatsziel Sozialstaatlichkeit unterschieden werden, vgl. Link, VVDStRL 48 (1990), 7, 18 und 27 ff. sowie Ress, VVDStRL 48 (1990), 56, 62 und 83. Kritisch zum Staatszweck Sicherheit Lepsius, Leviathan 32 (2004), 64, 88.

3 Ähnlich Pitschas, JZ 1993, 857; zum sozialstaatlichen Paradigma siehe Volkmann, JZ 2004, 696, $698 \mathrm{ff} .$.

4 Siehe dazu den aktuellen Bundeshaushalt 2005, der alleine für das Arbeits- und Sozialministerium Ausgaben in Höhe von ca. 80 Mrd. € vorsieht, mithin knapp ein Drittel des Gesamthaushaltes, vgl. www.bundesfinanzministerium.de. 
die für den Bürger materiell spürbarste und damit, so die hier vertretene These, faktisch bedeutsamste Form staatlicher Legitimation.

Jegliche Bemühungen zur Reformierung einer Solidargemeinschaft, die ihre Wohltaten mittel- und langfristig nicht mehr bezahlen kann, laufen zwangsläufig auf Kürzungen sozialer Zuwendungen hinaus. Dieser Umstand legt zumindest die Vermutung nahe, dass mit der damit einhergehenden Enttäuschung der an den Sozialstaat gerichteten Erwartungshaltung des Bürgers gegenüber dem seine Fürsorgeleistungen reduzierenden und die Maschen des sozialen Netzes weiter knüpfenden Staat dieser seine derzeit evidenteste Legitimation aufs Spiel setzt, mithin also eine Entwicklung einleitet, an deren Ende sich eine veritable, wenn auch in ihren Grenzen schwer zu vermessende staatliche Legitimationslücke auftun könnte. ${ }^{5}$

Das Schließen dieser Lücke lässt sich nun im Wesentlichen auf zwei verschiedenen Wegen denken: entweder durch eine - wie es etwa den skandinavischen Ländern gelungen zu sein scheint ${ }^{6}$ - dem Bürger plausibel gemachte Neudefinition des Sozialstaates im Sinne einer im Vergleich zum status quo eingeschränkten, ihre Defizite durch bürgerliches Engagement ausgleichenden Leistungsverwaltung oder aber, indem der Blick des Bürgers auf einen anderen, für diesen ebenso akzeptablen identitätsstiftenden Staatszweck gelenkt wird. Soweit ersichtlich versuchen die politischen Akteure in Deutschland derzeit, gleichzeitig beide Ansätze zu verfolgen. So lässt sich einerseits seit Jahren eine Strategie identifizieren, die den Begriff des Sozialstaats neu definiert, indem sie den politisch vorherrschenden Staatskonzeptionen des schlanken ${ }^{7}$ bzw. des aktivierenden ${ }^{8}$ Staates folgend den Einzelnen auffordert, sich in seinem Selbstverständnis von der Rolle des ausschließlich passiven (und sich gelegentlich dem Vorwurf des Hedonismus ausgesetzt sehenden) Versorgungsempfängers zu lösen, und die gleichzeitig den Begriff der Eigenverantwortung, ${ }^{9}$ der dem Staat einen Rückzug auf eine Grundversorgung gestattet, in den Mittelpunkt rückt. ${ }^{10}$ Andererseits sind - politisch bisher eher hinter vorgehaltener Hand diskutierte - Tendenzen erkennbar, die auf eine Ablösung der Vorherrschaft des Sozialstaates durch die eines den ak-

5 In diesem Sinne auch Wallerath, JZ 2004, 949; Düx, ZRP 2003, 189, 192; Zacher, Stimmen der Zeit 2001, 363, 375. Dabei gilt es allerdings das Phänomen zu bedenken, dass die Inanspruchnahme sozialer Leistungen mit einer Indifferenz gegenüber dem Sozialstaatsprinzip einhergehen kann, vgl. Nolte, Generation Reform, 4. Auflage 2004, S. 70 f..

6 Vgl. etwa Perger, Die Zeit 45/2003, S. 8.

7 Bestandteil der Programmatik der Regierung Kohl, vgl. Benz, Die Verwaltung 1995, 337, 341.

8 Der aktivierende Staat ist das Leitbild der noch amtierenden Bundesregierung, vgl. www.staat-modern.de; Trube/Wohlfahrt, SozArb 53 (2004), 2 f.; Dahme/Wohlfahrt, Neue Praxis 2002, 10 ff.; Dehnhard, RuP 2001, 45 ff.; kritisch Franzius, Der Staat 42 (2003), 493 ff.; zur Konzeption des kooperativen Staates als weiterem Denkmodell siehe grundlegend Ritter, AöR 104 (1979), 389 ff..

$9 \mathrm{Zu}$ den Dimensionen des Begriffs Eigenverantwortung siehe Trenk-Hinterberger, in: FS Zacher, 1998, S. 1163 ff..

10 Dabei gilt es allerdings zu berücksichtigen, dass das geltende Sozialversicherungsrecht nur sehr begrenzte Möglichkeiten selbstverantwortlichen Handelns eröffnet, vgl. Schnapp, DVB1 2004, 1053, 1061. 
tuellen Staatskonzeptionen zumindest prima vista zuwiderlaufenden ${ }^{11}$ Präventionsstaates in erster Linie der inneren Sicherheit hinzielen. ${ }^{12}$

Diese Entwicklungslinien kennzeichnen damit einen Paradigmenwechsel, der geeignet ist, das Grundverständnis unseres Gemeinwesens zu verändern. Wurde das Phänomen des Präventionsstaates bisher weitgehend vor dem Hintergrund seiner befürchteten Unvereinbarkeit mit dem liberalen Rechtsstaat diskutiert, skizziert der vorliegende Beitrag also eine andere Konfliktlinie: die des Verhältnisses zwischen dem sozialen Rechtsstaat ${ }^{13}$ und dem Präventionsstaat im Sinne einer Konkurrenz um die Position des dominanten Staatszweckes. Dabei handelt es sich zwar nicht um einen reinen Antagonismus, da das Sicherheitsrecht als zentrales Element des Präventionsstaates teleologisch auch vom Sozialstaatsprinzip aufgeladen wird. ${ }^{14}$ Andererseits wohnt diesem Umstand keinerlei den oben genannten Konflikt lösende Wirkung inne.

Es ließe sich in diesem Zusammenhang auch eine erheblich weitere, maßgeblich von der Präventionsdiskussion im Umweltschutzrecht inspirierte Perspektive wählen, die eine Ablösung des Sozialstaates durch einen über die innere Sicherheit hinaus reichenden ganz allgemeinen Vorsorgestaat postuliert. ${ }^{15}$ In der Tat ist ein derart umfassender Präventionsstaat als Instrument zur Steuerung einer nicht nur in ihrer inneren Sicherheit bedrohten Risikogesellschaft ${ }^{16}$ konstruierbar, die sich von individuellen Verantwortungen zunehmend verabschiedet. Diese Entwicklung lässt sich zudem unmittelbar vom Sozialstaat ausgehend denken, wenn man nämlich annimmt, dass sich der soziale Staat im Rahmen seines Umbaus weg von der materiellen individualisierten hin zur kollektivierten Fürsorge immer mehr Lebensbereiche erschließt und letztlich in der umfassenden Risikovorsorge eines Präventionsstaates endet. ${ }^{17}$ Die Kompatibilität eines solchen Staatsverständnisses mit den Konzepten eines schlanken oder aktivierenden Staates bleibt allerdings vollends unklar. Darüber hinaus ist die Frage,

11 Siehe aber Kreissl, in: Stüllenberg (Hrsg.), Zukunftsstudie Organisation von Sicherheit in Deutschland 2013, S. 35, 48; Dahme/Wohlfahrt, Neue Praxis 2002, 10, 24 ff..

12 Düx, ZRP 2003, 189, 192; Sack, in: Roggan (Hrsg.), Sicherheit vor Freiheit?, 2003, S. 59, 69 f.; ders., in: Dahme/Trube/Otto/Wohlfahrt (Hrsg.), Soziale Arbeit für den aktivierenden Staat, 2003, S. 249, 251 f.; Miegel, Die deformierte Gesellschaft, 2002, S. 261; Lange/ Schenck, Polizei im kooperativen Staat, 2002, S. 368 f.; siehe auch Buckel/Kannankulam, Das Argument 44 (2002), 34, 37; relativierend P.-A. Albrecht, Kriminologie, 2. Auflage 2002, S. $70 \mathrm{ff} .$.

13 Auf die traditionelle Auseinandersetzung zwischen liberalem und sozialem Rechtsstaat sei an dieser Stelle zumindest hingewiesen, vgl. etwa Zacher, in: Isensee/Kirchhof (Hrsg.), HdbStR I, 2.Auflage 1995, § 25 Rn 95. Der vorliegende Beitrag setzt damit gleichsam zwei Konkurrenzinstitute des liberalen Rechtsstaates zueinander in Beziehung.

14 Möstl, Die staatliche Garantie für die öffentliche Sicherheit und Ordnung, 2002, S. 19 ff..

15 Volkmann, JZ 2004, 696, 700 f.; siehe auch Denninger, KJ 1988, 1, 14 f..

16 Hierzu begriffsbildend Beck, Risikogesellschaft, 1986, S. 26; siehe aber Kötter, Der Staat 43 (2004), 371, 375; Albers, Die Determination polizeilicher Tätigkeit in den Bereichen der Straftatenverhütung und der Verfolgungsvorsorge, 2001, S. 97 Fn 2. Zu Begriff und Inhalt des Risikoverwaltungsrechts siehe Scherzberg, VVDStRL 63 (2004), 216 ff.; Lepsius, VVDStRL 63 (2004), 264 ff.; Pitschas, DÖV 2002, 221, 229.

17 Siehe dazu Hesse, Der Schutzstaat, 1994, S. 115 ff.; Aulehner, Polizeiliche Gefahren- und Informationsvorsorge, 1998, S. 328. 
inwieweit in einem solchen Vorsorgestaat klassische sozialstaatliche Elemente der individuellen Sicherung noch ihren Platz haben können, zumindest tendenziell zu verneinen. Auch diese Staatsperspektive rechtfertigt daher die Annahme einer Dominanz der inneren Sicherheit.

\section{Zum Begriff des Präventionsstaates}

Für den Präventionsstaat fehlt es bislang offenbar an einer einheitlichen und konsentierten Definition. Versucht man den Begriff zunächst thematisch zu verorten, so findet sich sein Schwerpunkt deutlich im Themenfeld der inneren Sicherheit. ${ }^{18}$ Festen Boden hat man damit allerdings noch nicht unter den Füßen, wird doch angesichts einer zusammenwachsenden Welt mit ihren globalisierten Gefahrenszenarien zunehmend die Frage erörtert, ob die Kategorisierung in innere und äußere Sicherheit nicht als überholt bezeichnet werden muss. ${ }^{19}$ Stellt man derartige Überlegungen hintan, so wird der Präventionsstaat wohl weitgehend als Staat verstanden, der die innere Sicherheit in einer von komplexen Problemlagen und wachsendem Steuerungsbedarf geprägten Gesellschaft ${ }^{20}$ dadurch aufrecht erhalten will, dass er Gefahren und Risiken im weitesten Sinne vorbeugend begegnet, indem er zu massiven, möglichst umfassenden Überwachungsmaßnahmen greift. ${ }^{21,22}$ Das Ziel also entspricht den staatlichen Schutzpflichten geradezu idealtypisch, die dazu eingesetzten Mittel hingegen bergen mannigfaltigen Konfliktstoff, konfligieren doch die Ziele des dem Kollektiv gewährten Schutzes durch den Staat einerseits und des Schutzes des Individuums vor dem Staat ${ }^{23}$ andererseits. Denninger etwa als einer der profiliertesten Kritiker des Präventionsstaates fasst zusammen, dass dieser Gefahr laufe, sich in seiner proaktiven Funktionslogik vom Grundsatz der Verhältnismäßigkeit abzuwenden und in der Maßlosigkeit zu verirren, da er sich nicht vom Prinzip der Rechtsgüterabwägung leiten lasse, sondern am Erfolg des Rechtsgüterschutzes orientiere. ${ }^{24}$

$18 \mathrm{Zu}$ weiteren Begriffsfeldern der Sicherheit Brugger, VVDStRL 63 (2004), 101, 121; siehe auch Volkmann, JZ 2004, 696, $700 \mathrm{f}$..

19 Vgl. Gusy, GA 2005, 214, 217; Isensee, in: Mellinghoff/Morgenthaler/Puhl, Die Erneuerung des Verfassungsstaates, 2003, S. 7, 12 ff.; Denninger, KritV 2003, 313, 314; Ahlf, Die Polizei 2002, 93 ff.; Möstl, Die staatliche Garantie für die öffentliche Sicherheit und Ordnung, 2002, S. 279 ff.; Erbel, Aus Politik und Zeitgeschichte 2002, B 10-11, 14, 16.

20 Vgl. die von P.-A. Albrecht, KritV 1986, 55, 82 geäußerte These, wonach der Weg zum Präventionsstaat über staatliche und gesellschaftliche Steuerungskrisen führt.

21 Umfassend zum Diskussionsstand Paeffgen, GA 2003, 648. Zum Potential für eine Totalüberwachung im Strafprozessrecht und Polizeirecht Wolter, in: FS Rudolphi, 2004, S. 733, 745.

22 Fraglich ist, ob der gerne gegebene Hinweis auf die im Vergleich zur staatlichen Überwachung ungleich umfangreichere Datenkumulation und -nutzung durch die freie Wirtschaft (vgl. Käppner, Süddeutsche Zeitung vom 18.05.2005, S. 4) eher zur Relativierung präventionsstaatlicher Bemühungen oder aber als warnendes Beispiel für die nahezu unbegrenzten Möglichkeiten umfassender Datenfremdherrschaft durch wen auch immer taugt.

23 Vgl. Schoch, Der Staat 43 (2004), 347, 363 ff..

24 Denninger, KJ 2002, 467, 472; ders., www.goethe.de/kug/ges/rch/thm/de; ders., begriffsbildend, teilweise aber noch aus anderer Perspektive, KJ 1988, 1 ff.; Prantl, Verdächtig, 
So berechtigt diese vor Missbrauch warnenden Hinweise sind, sollte doch insbesondere vor einer vorschnellen Gleichsetzung des Präventionsstaates mit einem Polizeistaat Abstand genommen werden. ${ }^{25,}{ }^{26}$ Der Polizeistaat ${ }^{27}$ wird üblicherweise definiert als System der Ausübung von Staatsgewalt im Innern ohne Bindung an Verfassung, parlamentarische Gesetzgebung, Gewaltenteilung, ohne Rücksicht auf private und bürgerliche Rechte und Freiheiten und ohne gerichtlichen Rechtsschutz. ${ }^{28}$ Trotz bedenklicher Entwicklungen vor allem im legislativen und exekutiven Detail namentlich in Form einer zunehmenden Ausweitung von Eingriffen in das Recht auf informationelle Selbstbestimmung erscheint aber kaum vorstellbar, dass unsere derzeitige staatliche Verfasstheit in absehbarer Zeit einer derartigen Degeneration unterliegen könnte. ${ }^{29}$ Insoweit sei nur auf die evidente Funktionsfähigkeit der Judikative im Allgemeinen und des BVerfG im Besonderen hingewiesen, das nicht nur einschlägige Grundsätze aufgestellt, ${ }^{30}$ sondern gerade in der jüngeren Vergangenheit auch verschiedentlich deutliche Korrekturen vorgenommen hat. ${ }^{31}$ Für die folgende Erörterung soll der Begriff des Polizeistaates als Parameter daher ausscheiden.

Soweit im Folgenden vom Präventionsstaat die Rede ist, soll ohnehin nicht von einem zu erwartenden oder befürchtenden Endzustand der Entwicklung die Rede sein, sondern statt dessen vom vorgefundenen, gegenwärtigen Stand der Dinge ausgegangen werden. Zu Grunde zu legen ist damit nach hier vertretener Auffassung ein bereits über den status nascendi hinausgelangter Präventionsstaat, der sich als ein erstens zunehmend auf Aspekte vor allem der inneren Sicherheit reduzierter, zweitens dabei aber ganzheitlich präventiver wie repressiver, auch die Prävention im klassischen polizeilichen Sinne verfolgenden Wohlfahrtsstaat beschreiben lässt. Der Präventionsstaat im hier verstandenen und diskutierten Sinne beruht auf einem grundsätzlich in den Rechts-

2002, S. 9 f.; zur fehlenden Abwägungsfähigkeit des Begriffes Sicherheit siehe auch Lepsius, Leviathan 32 (2004), 64, 87 f.; explizit positiv zum Präventionsstaat Tettinger, in: FS Kirchhoff, 2002, S. 281, 290.

25 Ähnliches gilt für Begrifflichkeiten wie Sicherheitsstaat (siehe dazu z.B. P.-A. Albrecht, Die vergessene Freiheit, 2003, S. 28 ff. mit empirischen Beispielen; zu Definition und Kritik des Sicherheitsstaates aus sozialistischer Sicht siehe Hirsch, Der Sicherheitsstaat, 1980, S. 94 ff. und 111 ff.) oder Überwachungsstaat. $\mathrm{Zu}$ noch anderen Formulierungen siehe Vormbaum, ZStW 107 (1995), 734, 740.

26 Ähnlich Schoch, Der Staat 43 (2004), 347, 365; Denninger, StV 2002, 96, 97.

27 Der Begriff des Wohlfahrtsstaates als synonyme Epochenbezeichnung zum Polizeistaat des 18. Jahrhunderts darf selbstverständlich nicht verwechselt werden mit dem der Wohlfahrtspflege innerhalb des Sozialstaates.

28 Götz, Allgemeines Polizei- und Ordnungsrecht, 13. Auflage 2001, Rn 8.

29 Anders, allerdings unter Zugrundelegung einer überzogenen Definition, Roggan, Auf legalem Weg in einen Polizeistaat, 2000, S. 23, der im hier diskutierten Perspektivwechsel von der Leistungs- zur Eingriffsverwaltung ein Kennzeichen des Polizeistaates erblickt.

30 Etwa zum Verbot der Totalüberwachung zuletzt BVerfG, 2 BvR 581/01 vom 12.04.2005, $60 \mathrm{ff}$, www.bverfg.de.

31 So z.B. in BVerfGE 100, $313 \mathrm{ff}$. zur Überwachung des Fernmeldeverkehrs, in BVerfGE 103, 142 ff. zur Gefahr im Verzug, in BVerfGE 103, 21 ff. zur DNA-Feststellung, in BVerfGE 109, 279 ff. zur akustischen Wohnraumüberwachung, und in BVerfGE 110, $33 \mathrm{ff}$. sowie jüngst in BVerfG, 1 BvR 668/04 vom 27.07.2005, www. bverfg.de zur präventiven TKÜ. Kritisch zur Rolle des BVerfG allerdings Arnold, StraFo 2005, 2 ff.. BvR BvR 
staat eingebetteten und seinen Prinzipien unterworfenen Staatsverständnis, innerhalb dessen allerdings die aktiven und passiven Verpflichtungen des Bürgers, nämlich einerseits zur eigenen Beitragsleistung in der inneren Sicherheit und andererseits zur Duldung von Rechtseingriffen neu bewertet und justiert werden. ${ }^{32}$ Fraglos ist im Rahmen dieses Prozesses auch immer wieder das Betreten bedenklichen, vor allem verfassungsrechtlich verminten Terrains zu befürchten, doch ändert dies nichts an dem Umstand, dass die Idee eines solchen Präventionsstaates an sich nicht zwangsläufig mit einer Verabschiedung des Rechtsstaates einhergehen muss, ${ }^{33}$ wenngleich zuzugeben ist, dass das Bemühen um Vereinbarkeit der Funktionslogik des Präventionsgedankens mit der des Rechtsstaates schwerwiegende Probleme aufwirft. Diese können nur gelöst werden, wenn die zur Verfolgung des Präventionszieles herangezogenen Mittel und Methoden konsequent am Grundsatz der Verhältnismäßigkeit ${ }^{34}$ gemessen werden, dessen ratio für den hier erörterten Problemkreis klassisch schon von Svarez ${ }^{35}$ beschrieben worden ist, dass nämlich der Staat die Freiheit des einzelnen nur soweit einzuschränken berechtigt sei, als es notwendig ist, damit die Sicherheit und Freiheit aller bestehen könne.

\section{Zur Entwicklung des Präventionsstaates}

Die von den Philosophen der frühen Neuzeit formulierte Idee, sich durch die Mitgliedschaft in einer Gemeinschaft und das Zugeständnis eines Gewaltmonopols des Gemeinwesens einen Anspruch auf persönlichen Schutz und Sicherheit zu erwerben, darf als raison d'être für die Entstehung von Staaten überhaupt gelten. ${ }^{36}$ Insoweit ist ein

32 So auch Schoch, Der Staat 43 (2004), 347, 366 ff; ähnlich, in der Gesamttendenz aber deutlich konservativer Horn, in: FS Schmitt Glaeser, 2003, S. 435, 457 ff. .

33 In diesem Sinne Schoch, Der Staat 43 (2004), 347, 361 und wohl auch Gusy, VVDStRL 63 (2004), 151, 179 f.; Pitschas, JZ 1993, 857, 858 spricht etwas kryptisch vom Präventionsstaat als »materialem Rechtsstaat«. A. A. Limbach, AnwB1 2002, 454, 457, die davon ausgeht, dass sich der Grenzverlauf zwischen einem Rechts- und einem Präventionsstaat nicht eindeutig markieren lasse, mithin damit aber eine Grenze annimmt; ähnlich Denninger, StV 2002, 96, 97; ders., KJ 2002, 467, 470 und 474.

34 Instruktiv zur Frage der Verhältnismäßigkeit von Prävention Grimm, KritV 1986, 38, 50 ff.. Calliess, ZRP 2002, 1, 6 ff. schlägt in diesem Zusammenhang ein Abwägungsmodell zwischen Freiheit und Sicherheit im Sinne einer Schnittmengentheorie vor; ihm folgend Horn, in: FS Schmitt Glaeser, 2003, S. 435, 450 f.; dagegen zu Recht kritisch Düx, ZRP 2003, 189, 194. Denninger, KJ 2002, 476, 470 weist auf die Notwendigkeit einer kohärenten, rechtsstaatlichen Maßstäben und Präventionsbedürfnissen gleichermaßen entsprechenden Sicherheitspolitik hin. Insgesamt skeptisch Volkmann, JZ 2004, 696, 702. Zur Aushebelung des Abwägungsprinzips siehe Gusy, VVDStRL 63 (2004), 151, 176 ff..

35 Svarez, in: Conrad/Kleinheyer (Hrsg.), Vorträge über Recht und Staat, 1960, S. 39.

36 Das BVerfG hat bekanntlich in den Zeiten des RAF-Terrors in E 49, 24, 56 f. unter Berufung auf BVerwGE 49, 202, 209 festgestellt, dass die Sicherheit des Staates als verfasster Friedensmacht und die von ihm zu gewährleistende Sicherheit seiner Bevölkerung ranghohe, unverzichtbare Verfassungswerte seien, weil die Institution Staat von ihnen die eigentliche und letzte Rechtfertigung herleite. Zum weiten Feld der staatstheoretischen Herleitung vgl. z.B. Brugger, VVDStRL 63 (2004), 101, 111 ff.; Volkmann, JZ 2004, 696, 697; Calliess, ZRP 2002, 1, 2 ff.; Kniesel, ZRP 1996, 482, 485; zur Frage eines Grundrechts auf Sicherheit Isensee, Grundrecht auf Sicherheit, 1983, insb. S. 33; Robbers, Sicherheit als Menschenrecht, 
Rekurs auf die Idee der durch den Staat gesellschaftsvertraglich vermittelten Sicherheit, die allerdings raumgreifend neu interpretiert wird, ${ }^{37}$ durchaus schlüssig, noch dazu in einer alternden ${ }^{38}$ und durch gesellschaftliche Veränderungsprozesse verunsicherten Bevölkerung. Dieser Rekurs erfolgt auf den Ebenen der Legislative und der Exekutive, während die Judikative eher um Korrekturen bemüht scheint. ${ }^{39}$

\section{Legislative}

Gelegentlich wird als Geburtsstunde des Präventionsstaates der 11.09.2001 diskutiert. ${ }^{40}$ Daran ist sicher richtig, dass der eingeleitete Paradigmenwechsel in den Staatszwecken gerade zu Beginn des 3. Jahrtausends im Lichte des vom Terroranschlag in New York ausgelösten internationalen sicherheitspolitischen Aktionismus und auf Grund der gleichzeitigen Bemühungen zur Revision des Sozialstaates besonders augenfällig geworden ist. Andererseits ist dem zunächst entgegenzuhalten, dass die zur Terrorismusbekämpfung gesammelten Maßnahmen ${ }^{41}$ in weiten Teilen bereits in die politische Diskussion eingeführt waren und der 11.09.2001 insoweit nur den Anlass gab, altbekannte, aber bis dahin nicht ohne weiteres durchsetzbare Forderungen zu realisieren. ${ }^{42}$ Insoweit hat dieses Datum lediglich zu einer schnelleren Beförderung des Gedankens des Präventionsstaates beigetragen. Darüber hinaus lassen sich die ersten Indizien für das Entstehen eines Präventionsstaates im hier diskutierten Sinne mindestens zu Beginn der 90er Jahre identifizieren. ${ }^{43}$ Zuvor war die Verschärfung des Polizei-, des Straf- und des Strafverfahrensrechts im Wesentlichen reaktiv, anlassbezogen und konkreten Bedrohungsszenarien bzw. historisch abgeschlossenen Vorgängen wie etwa dem Terrorismus der 70er Jahre oder dem Demonstrationsgeschehen in den 80er Jahren geschuldet, aber im Übrigen immer wieder isoliert stehen geblieben ${ }^{44} \mathrm{Zu}$ Beginn der darauf folgenden

1987; Brugger, VVDStRL 63 (2004), 101, 129 ff.; Gusy, VVDStRL 63 (2004), 151, 168 ff.; Paeffgen, GA 2003, 648, 659; Limbach, AnwBl 2002, 454, 455; Hassemer, Freundesgabe Büllesbach, 2002, S. 225, 232; Aulehner, Polizeiliche Gefahren- und Informationsvorsorge, 1998, S. 428 ff. Beachte auch Art. 99 S. 2 der Bayerischen Verfassung.

37 Vgl. Kötter, KJ 2003, 64, 76 f..

38 Allerdings gilt es zu bedenken, dass eine vergreisende und deshalb weniger delinquente Gesellschaft die objektive innere Sicherheit im Grunde genommen eher befördert.

$39 \mathrm{Vgl}$. Fn 31. Zur gleichzeitigen Aufgabe der Rechtsprechung, den Abbau sozialer Leistungen zu begrenzen, vgl. jüngst BVerfG, 1 BvR 1508/96 vom 07.06.2005, 35 ff., www.bverfg.de.

40 Z.B. bei Prantl, AnwBl 2002, 274.

41 Einen aktuellen Überblick über die seither gemachten legislativen Anstrengungen auf nationaler und internationaler Ebene gibt Saurer, NVwZ 2005, 275 ff..

42 Vgl. Hetzer, ZRP 2005, 132, 134; P.-A. Albrecht, Die vergessene Freiheit, 2003, S. 17 f.; Hoffmann-Riem, ZRP 2002, 497, 498; Hirsch, Vorgänge 3/2002, 5, 6. Zu den Aktivitäten auf Ebene der europäischen Antiterrorpolitik Gusy, GA 2005, $214 \mathrm{ff..}$

43 Vgl. Denninger, StV 2002, 96; Hassemer, Vorgänge 3/2002, 10, 11; Kniesel, ZRP 1996, 482.

44 Inwieweit dies hinsichtlich der Bekämpfung des RAF-Terrors lediglich wegen der damals vor allem technisch noch begrenzteren Möglichkeiten gilt, mag dahinstehen. Prantl, Verdächtig, 2002, S. 10 sieht schon in der RAF-Zeit die Vorboten des Präventionsstaates; ähnlich Paeffgen, GA 2003, 648, 652; Düx, ZRP 2003, 189. Gusy, VVDStRL 63 (2004), 151, 182 hingegen sieht die Sicherheitsdiskussion der vergangenen Jahrzehnte von einer Abfolge von trial and error geprägt. 
Dekade hingegen begannen verschiedene scheinbar nicht im Zusammenhang stehende, sich in der Zusammenschau aber ergänzende und allesamt konsequent in Richtung eines Präventionsstaates zielende Entwicklungen. Für einen ersten großen Anschub hin zum Präventionsstaat sorgten spätestens die mit dem Anwachsen der organisierten ${ }^{45}$ und der grenzüberschreitenden Kriminalität angesichts des Falls des eisernen Vorhangs und des europäischen Einigungsprozesses Anfang der neunziger Jahre einhergehenden legislativen Bekämpfungsstrategien, die bis heute sukzessive vor allem zu einer Ausdehnung des polizeilichen ${ }^{46}$ - hier wurde den hergebrachten Begriffen der konkreten Gefahr und des Störers der Abschied gegeben und damit die traditionellen Strukturen des Gefahrenabwehrrechts durchbrochen ${ }^{47}$ - und strafprozessualen ${ }^{48}$ Befugnisspektrums nebst einer zunehmenden Auflösung der zwischen diesen beiden Polen gezogenen Grenzen führten. ${ }^{49}$

\section{Exekutive}

Die von der Legislative bereitgestellten Eingriffswerkzeuge wurden und werden von der Exekutive, die diese vielfach auch nachgefragt hatte, in Gebrauch genommen. Gleichzeitig betont sie aber auch die Notwendigkeit all dessen, was sich - wenigstens in Teilen eingriffsneutral und der Kritik am Präventionsstaat daher kaum ausgesetzt ${ }^{50}$ - im Bereich der hergebrachten Kriminalprävention manifestiert. So enthält die 1994 erfolgte Fortschreibung des Programms Innere Sicherheit von $1974^{51}$ neben etlichen repressiv orientierten Forderungen durchaus auch zahlreiche Aspekte der klassischen polizeilichen Prävention und daneben der Bürgerbeteiligung, um neben der formellen polizeilichen auch die informelle Sozialkontrolle zu aktivieren. Seit den 90er Jahren erfolgt zudem eine intensive Befassung mit polizeilichen Strategien vor allem der USA, deren (vermeintliche) Bandbreite vom zero tolerance-Ansatz bis hin zum community policing reicht. Auf diese Weise wurde die Prävention vor allem unter dem Stichwort der Kommunalen Kriminalprävention im Zuständigkeitsbereich bürgerlicher und gesamtgesellschaftlicher Selbstverantwortung verortet und hat in den vergangenen Jahren an Bedeutung gewonnen. ${ }^{52}$ Gerade dieser Aspekt wird von den Kriti-

45 Dazu Hefendehl, StV 2005, 156 ff.; Frehsee, Der Rechtsstaat verschwindet, 2003, S. 291 f..

46 Als Stichworte sind unter anderem zu nennen Schleierfahndung, präventive Videoüberwachung, präventive Wohnraumüberwachung, präventive Rasterfahndung und jüngst präventive TKÜ und DNA-Analyse.

47 Vgl. Schulze-Fielitz, in: FS Schmitt Glaeser, 2003, S. 407, 409 ff..

48 Hier finden sich, nicht zuletzt in logischem Zusammenhang mit der steten Zunahme abstrakter Gefährdungsdelikte im Strafrecht (dazu Frehsee, Der Rechtsstaat verschwindet, 2003, S. 278 f.), weitgehend die strafprozessualen Pendants zu den Verschärfungen des Polizeirechts.

49 Siehe insgesamt Kötter, KJ 2003, 64, 65 f.; Hirsch, Vorgänge 3/2002, 5 , 7 f..

50 Vgl. aber Sack, in: Gössner (Hrsg.), Mythos Sicherheit, 1995, S. 429 ff..

51 Programm Innere Sicherheit, Fortschreibung 1994 durch die Innenminister/-senatoren der Länder und den Bundesminister des Innern; siehe auch den Beschluss der IMK vom 2.2.1998 zu einer »Partnerschaft für mehr Sicherheit in unseren Städten und Gemeinden«.

52 Kötter, Der Staat 43 (2004), 371, 379 ff.; siehe nur die umfassenden Dokumentationen des BKA unter www.bka.de/vorbeugung/infopool bzw. /linksammlung. Unter den vielfältigen sonstigen Aktivitäten sind vor allem der Deutsche Präventionstag oder die 2001 gegründete Stiftung Deutsches Forum für Kriminalprävention hervorzuheben. 
kern des Präventionsstaates nicht immer hinreichend gewürdigt. ${ }^{53}$ Andere Wege des Engagements Privater zur Aufrechterhaltung der öffentlichen Sicherheit und Ordnung sind mit Modellen des bürgerlichen Engagements wie der bayerischen Sicherheitswacht und vor allem dem Einsatz privater Sicherheitsdienste ${ }^{54,55}$ beschritten worden. Schließlich ist unter der Überschrift Exekutive darauf hinzuweisen, dass seit den 90er Jahren verschiedenste Aktivitäten zur Reorganisation der Polizeien auf Bundes- wie auf Landesebene zu verzeichnen sind ${ }^{56}$ die aktuell in eine Diskussion um eine neue Sicherheitsarchitektur in Deutschland und darüber hinaus ${ }^{57}$ einschließlich einer Infragestellung des Trennungsprinzips münden ${ }^{58}$ und auch den Einsatz der Bundeswehr im Innern erwägen. ${ }^{59}$

\section{Zwischenergebnis}

Alles in allem bietet sich so das Bild eines Staates, der in umfänglicher Weise um die Aufrechterhaltung der öffentlichen Sicherheit und Ordnung bemüht ist und dazu alle ihm zur Verfügung stehenden Register zieht, indem er einerseits die eigenen Kompetenzen hierzu konsequent ausbaut, andererseits aber auch die Gesellschaft in die Pflicht nimmt. Es mag letztlich dahin stehen, ob diese Entwicklung tatsächlich von vorneherein bewusst mit Blick auf den Niedergang des Sozialstaates eingeleitet worden ist oder zunächst zufälliger Natur war. Festzuhalten ist, dass sie als Gegenmodell zum bisher vorherrschenden Staatszweck zunehmend Konturen annimmt und damit politische Hoffnungen stützt, mit der Rückkehr zu Hobbes eine etwaige vom Niedergang des Sozialstaats gerissene Legitimationslücke zu schließen.

53 Siehe aber P.-A. Albrecht, KritV 1986, 55, $61 \mathrm{ff.}$.

54 V. Elsbergen fasst die einschlägigen Phänomene unter dem Begriff der Kustodialisierung zusammen, vgl. ders. (Hrsg.), in: Wachen, kontrollieren, patrouillieren - Kustodialisierung der inneren Sicherheit, 2004, S. $14 \mathrm{ff} .$.

55 Pitschas, Polizei und Sicherheitsgewerbe, 2000, S. 127 ff., postuliert in diesem Zusammenhang ein »sicherheitspolitisches Mitwirkungsverhältnis«.

56 Einen Überblick bietet der Bericht der Scheu-Kommission zur Neuorganisation der Polizeibehörden in Nordrhein-Westfalen, S. 41 ff., www.polizei.nrw.de.

57 Vgl. Saurer, NVwZ 2005, 275, 280; Hetzer, MSchrKrim 2005, 111, 114; Weidenfeld (Hrsg.), Herausforderung Terrorismus, 2004; Timm, Kriminalistik 2003, 203; Ahlf, Die Polizei 2002, 93, 97 ff.; Möstl, Die staatliche Garantie für die öffentliche Sicherheit und Ordnung, 2002, S. 509 ff.; hinzuweisen ist z.B. auf das seit Dezember 2004 in Berlin eingerichtete Gemeinsame Terrorabwehrzentrum von Bundeskriminalamt und Bundesamt für Verfassungsschutz (siehe dazu Würz, Kriminalistik 2005, 10 ff.).

58 Vgl. Gusy, VVDStRL 63 (2004), 151, 186; Nehm, NJW 2004, 3289 ff..

59 Vgl. Soria, DVBl 2004, 597 ff.; Lange, AöR 129 (2004), 459 ff.; Weidenfeld (Hrsg.), Herausforderung Terrorismus, 2004; umfassend nunmehr Fiebig, Der Einsatz der Bundeswehr im Innern, 2004; vgl. auch die aktuelle Diskussion um das Erfordernis einer Grundgesetzänderung im Kontext des LuftsicherheitsG, BGB1 I 2005, 78 ff. (dazu siehe Hartleb, NJW 2005,1397 ff; Kersten, NVwZ 2005, 661 ff.). 


\section{Präventionsstaat versus Sozialstaat}

Zunächst lässt sich nicht bestreiten, dass ein Präventionsstaat im hier diskutierten Sinne ein Grundbedürfnis der Menschen bedient. Schon deshalb muss das Streben eine Staates nach Sicherheit für seine Bürgerinnen und Bürger als legitim, da ihn schlechthin legitimierend betrachtet werden. Unter Berücksichtigung, aber auch jenseits der üblicherweise vorgebrachten prinzipiellen Kritik am Präventionsstaat in seiner nicht immer zu leugnenden Frontstellung zum liberalen Rechtsstaat wird sich ein das Paradigma Sozialstaat ablösender Präventionsstaat jedoch der Frage stellen müssen, ob er den Sozialstaat erstens in der politischen Realität und zweitens im Bewusstsein der Bürger abzulösen geeignet ist. Letztlich geht es um die pragmatische politische Prognose, ob der Bevölkerung im Rahmen des Vollzugs der Staatsaufgabe Sicherheit ${ }^{60}$ deren innere Ausformung mehr wert ist und sein kann als deren soziale. Bei den im Folgenden vertretenen Thesen zu diesem - sich in evidenten Widerspruch zum Diktum, dass eigentlich gerade eine gute Sozialpolitik die beste Kriminalpolitik sein sollte, ${ }^{61}$ setzenden - Unterfangen scheinen allerdings die im verfassungsrechtlichen Diskurs gebräuchlichen kritischen Argumente immer wieder durch.

\section{Sicherheit als Versorgungsgut}

Festzuhalten ist zunächst, dass der wissenschaftlich erst spät erschlossene Begriff der Sicherheit $^{62}$ als gemeinsamer Ausgangspunkt beider Dimensionen sowohl an seiner inhaltlichen Weite als auch - damit korrelierend - an seiner inflationären Verwendung leidet. Eine Annäherung vom Gegenpol der Bedrohung aus stößt auf ähnliche Schwierigkeiten. ${ }^{63}$ Der Mangel des Fehlens fester definitorischer Grenzen setzt sich beim in Rede stehenden Begriffspaar der inneren ${ }^{64}$ und sozialen ${ }^{65}$ Sicherheit fort. Auch die Gemeinsamkeit beider Termini, sich durch phänomenologische Konkretisierungen

60 Dazu Götz, in: Isensee/Kirchhof, HdbStR III, 2. Auflage 1996, § 79 Rn 1.

61 V. Liszt, Strafrechtliche Aufsätze und Vorträge, Band 2, 1905, S. 246.

62 Vgl. Holst, Sicherheit und Bedrohung, 1998, S. 7 ff.; Kaufmann, Sicherheit als soziologisches und sozialpolitisches Problem, 2. Auflage 1973.

63 Zudem reagiert die Bevölkerung auf Bedrohungslagen nicht einheitlich: die Gefahren des Straßenverkehrs beispielsweise sind trotz hohen Blutzolls gesellschaftlich ganz offenbar akzeptiert, vgl. Hoffmann-Riem, ZRP 2002, 497, 501; Schubarth, in: Bauhofer/Bolle, Innere Sicherheit - Innere Unsicherheit?, 1995, S. 153 ff..

$64 \mathrm{Zu}$ den verschiedenen Forschungsdisziplinen, die sich den Begriff der inneren Sicherheit erschließen, vgl. Lange, Innere Sicherheit im politischen System der Bundesrepublik Deutschland, 1999, S. 25 ff.; zur Definition siehe auch Erbel, Aus Politik und Zeitgeschichte 2002, B 10-11, S. 14, 16; Albers, Die Determination polizeilicher Tätigkeit in den Bereichen der Straftatenverhütung und der Verfolgungsvorsorge, 2001, S. 99; Kniesel, ZRP 1996, 482 ff.; Kunz, in: Bauhofer/Bolle, Innere Sicherheit - Innere Unsicherheit?, 1995, S. 327, 340; Bull, Zeitschrift für Sozialwissenschaft 1984, 153, 165 ff..

65 Die soziale Sicherheit ist, dem Begriff des Sozialstaats als Staatsziel, für den es eine erschöpfende, schulmäßige Definition nicht gibt und auch nicht geben kann, entsprechend ein offener und verfassungspolitischer Topos (vgl. Degenhart, Staaatsrecht I, 20. Auflage 2004, S. 175), zu dessen Ausfüllung das BVerfG dem Gesetzgeber einen weiten Spielraum eröffnet hat, vgl. BVerfGE 100, 271, 284. 
ebenso beschreiben zu lassen wie als rechtliche Prinzipienbegriffe oder aber akteursbezogen als Politikfeld, hilft nicht weiter: diese Kategorisierungen sind ihrerseits inhaltlich offen. ${ }^{66}$ Am ehesten gelingt noch eine Bestimmung der jeweiligen Kernbereiche: im Falle der sozialen Sicherheit ist dies die Absicherung des Existenzminimums durch Sozialversicherungen und Sozialhilfe, bei der inneren Sicherheit die Durchsetzung des staatlichen Gewaltmonopols zum Schutze der von der öffentlichen Sicherheit im Sinne des Polizeirechts umfassten Rechtsgüter. ${ }^{67}$

Auf dieser Basis steht der Sozialstaat, nach Bedürftigkeiten gestaffelt, im Grundsatz allen Bürgern mit letztlich finanziell messbaren individualisierten Unterstützungsleistungen zur Verfügung. Vollkommen durch das Netz des Sozialstaates fiel bislang nur, wer sich diesem verweigerte. Obdachlosigkeit ${ }^{68}$ beispielsweise ist, ohne die darin liegende individuelle Tragik verharmlosen zu wollen, in unserem Gemeinwesen im Grundsatz letztlich ein selbst gewähltes, Angebote zur Unterbringung ausschlagendes Schicksal. Ähnlich konkretisierte Gaben kann ein Präventionsstaat nicht verteilen. Weder lässt sich innere Sicherheit als Pauschalgröße ohne weiteres messen, ${ }^{69}$ erst recht nicht vor dem Hintergrund der Problematik des Sicherheitsgefühls, ${ }^{70}$ das sich objektiven oder logischen Maßstäben weitgehend entzieht, noch lässt sich selbst unter Berücksichtigung des im Einzelfall bestehenden Anspruchs auf polizeiliches Handeln, ${ }^{71}$ der sich in der Regel nur auf ermessensfehlerfreies Entscheiden beschränkt,

66 Dass eine abschließende Definition des Begriffs der inneren Sicherheit nicht gelingen mag, ist im Grunde genommen schon deswegen nicht weiter verwunderlich, weil auch für kontextuelle Teilbegriffe wie namentlich die organisierte Kriminalität (vgl. Hefendehl, StV 2005, 156, 157) oder den Terrorismus (vgl. Lutz, in: Koch (Hrsg.), Terrorismus - Rechtsfragen der äußeren und inneren Sicherheit, 2002, S. 9, 10 ff.) gilt, dass sie sich eindeutiger Definitionen entziehen.

67 Zum Recht auf Sicherung des Existenzminimums einerseits siehe Martinez Soria, JZ 2005, 644 ff., zur Durchsetzung des staatlichen Gewaltmonopols andererseits siehe Möstl, Die staatliche Garantie für die öffentliche Sicherheit und Ordnung, 2002, S. 127 f., der zutreffend die Deckungsgleichheit der Begriffe innere bzw. öffentliche Sicherheit herausstellt und durch den Rekurs auf hergebrachte Schutzgüter des Polizeirechts wenigstens dem Begriffskern der inneren Sicherheit in juristischer Hinsicht feste Konturen verleiht; ihm folgend Horn, in: FS Schmitt Glaeser, 2003, 435, 443; relativierend Isensee, Grundrecht auf Sicherheit, 1983, S. 23.

68 Im Übrigen ein typisches Problem an der Schnittstelle von Sozial- und Gefahrenabwehrrecht.

69 Die beliebte Streitfrage um die Messbarkeit von Prävention lässt sich bekanntlich durchaus bejahen, allerdings nur auf die Bewältigung konkreter Gefahrensituationen bezogen; der Präventionsstaat hingegen operiert ja in deren Vorfeld.

70 Zur Kritik an der üblichen Differenzierung zwischen objektiver und subjektiver Sicherheit und mit einem Plädoyer für Sicherheit als grundsätzlich subjektiv determinierten Begriff siehe Gusy, VVDStRL 63 (2004), 151, 159 f. und 182; Ahlf, Die Polizei 2002, 93, 96; Aulehner, Polizeiliche Gefahren- und Informationsvorsorge, 1998, S. 283. Kritisch zur Messung von Kriminalitätsfurcht Kury/Lichtblau/Neumaier, Kriminalistik 2004, 457 ff.; Überlegungen zur staatlichen Pflege des Sicherheitsgefühls stellt Schulze-Fielitz, in: FS Schmitt Glaeser, 2003, S. 407, 433 an.

71 Siehe z.B. Schenke, Polizei- und Ordnungsrecht, 2. Auflage 2003, Rn 104; Calliess, ZRP 2002, 1, 6. Ähnliches gilt auch für die soziale Sicherheit, die aus dem Sozialstaatsprinzip heraus nur im Einzelfall subjektive Rechte gewährt, vgl. Jarass/Pieroth, GG, 6. Auflage 2002, Art. 20 Rn 83; BVerfGE 82, 60, 80; BVerwGE 82, 364, 368. 
eine nach sozialstaatlichem Muster zu ermittelnde und befriedigende individuelle Bedürftigkeit hinsichtlich des zu gewährenden Gutes Sicherheit bestimmen. Der Präventionsstaat wird sich daher, selbst wenn er das Versorgungsgut der inneren Sicherheit in den Mittelpunkt rückt, im Grundsatz mit einer schon jetzt bestehenden gleichförmigen Grundversorgung eines abstrakten und nicht individualisierbaren, sondern vielmehr kollektivierten, jedenfalls aber diffusen Gutes etwa im Sinne eines »Klimas der Sicherheit in der Gesellschaft « bescheiden müssen. Nun soll diese Leistung nicht kleingeredet werden. Die Bewahrung eines solchen - allerdings kaum alleine von Sicherheitsbehörden produzierbaren - gerade in einer medial gesteuerten Welt ${ }^{72}$ anfälligen und manipulierbaren Gesellschaftsklimas ist eine kaum zu überschätzende Leistung und von maßgeblicher Bedeutung für die Funktionsfähigkeit eines Staates. ${ }^{73}$ Sie markiert allerdings auch die Grenze des Machbaren. Ein Präventionsstaat aber, dessen für den Bürger spürbaren positiven Effekte auf die Sicherheitslage ohne mediale Manipulation nicht über den status quo hinausreichen, verfügt damit auch nicht über ein Tauschgut, das er dem Bürger zur Kompensation für dessen Einbußen an sozialen Leistungen anbieten kann. ${ }^{74}$ Ein Argument für einen Paradigmenwechsel lässt sich folglich daraus kaum ableiten.

\section{Sicherheit als Garantie}

Ein Wesensmerkmal des Sozialstaats ist - erinnert sei nur an Blüms Wort von den sicheren Renten - eine prinzipielle Garantie von Mindeststandards, wenngleich zuzugeben ist, dass deren Einlösung ihm zunehmend schwer fällt. Ungeachtet des vagen Umfanges des sozialstaatlichen Leistungskatalogs lässt sich eine im Existenzminimum konkretisierende unterste Grenze identifizieren. Ein Staat, der selbst betont, dass sein Metier in erster Linie die innere Sicherheit ist, wird sich fragen lassen müssen, inwieweit er in vergleichbarer Weise eine Garantie wenigstens auf die oben beschriebene Grundversorgung geben kann. ${ }^{75}$ Diese Frage wird auch von einem Präventionsstaat legitimerweise nicht bejaht werden können. Andernfalls müsste er nämlich seinem Gewaltmonopol zwangsläufig auch noch ein Sicherheitsmonopol folgen lassen. Ist aber

$72 \mathrm{Zu}$ den besorgniserregenden Folgen der Wirkung von Medien auf Kriminalitätswahrnehmung, Strafbedürfnisse und Kriminalpolitik siehe Pfeiffer/Windzio/Kleimann, MschrKrim 2004, 415 ff.; zu Kriminalitätsdarstellungen in den Massenmedien siehe die Beiträge in Kania/Walter/H.-J. Albrecht (Hrsg.), Alltagsvorstellungen von Kriminalität, 2004, S. 117 ff.; zur Darstellung von Risiken in den Medien siehe Schütz/Peters, Aus Politik und Zeitgeschichte 2002, B 10-11, 40 ff..

73 Und kann im Übrigen im Einzelfall auch sozialpolitische Effekte zeitigen, vgl. Möstl, Die staatliche Garantie für die öffentliche Sicherheit und Ordnung, 2002, S. 20 f..

74 Soweit in diesem Zusammenhang eingewandt wird, innere Sicherheit sei in gewissem Sinne auch deshalb Versorgungsgut, weil sie als Wirtschaftsfaktor von hoher Bedeutung sei, erscheint das für das oben beschriebene »Klima« der inneren Sicherheit durchaus plausibel. Zu dessen Gewährleistung bedarf es allerdings wie gezeigt eben keines besonderen Präventionsstaates, der in seiner Regelungs- und Überwachungsdichte zudem eher wirtschaftsfeindliche Effekte zeitigen würde.

75 Glaeßner, Aus Politik und Zeitgeschichte 2002, B 10-11, 3, 5 spricht von Reduktion von Unsicherheit statt von Garantie von Sicherheit. 
bereits das Gewaltmonopol nach richtiger Ansicht im Sinne eines Bestimmungsrechts über Ausübungsbefugnisse legitimer Gewalt und nicht im Sinne eines Monopols der Rechtsdurchsetzung zu verstehen, ${ }^{76}$ und folgt daraus die Zulässigkeit der Einbeziehung Privater in die Bewältigung dieses Auftrags, ${ }^{77}$ bleibt für ein Sicherheitsmonopol erst recht kein Raum mehr. ${ }^{78}$ Nach dem oben zu Grunde gelegten Verständnis vom Präventionsstaat ist das auch nicht bedenklich, wenngleich es für die Privatisierung von Sicherheit schon deswegen festgelegter Rahmenbedingungen ${ }^{79}$ bedarf, weil sich Maßnahmen nichtimperativer Steuerung rechtsstaatlichen Sicherungen tendenziell entziehen. ${ }^{80}$ Unmittelbar dem Staat zuzurechende Sicherheitsgarantien auch auf niedrigem Niveau lassen sich angesichts des Fortschreitens derartigen Outsourcings aber zunehmend nicht mehr identifizieren.

Gilt es, solche Garantien zu vermeiden, so verlockt der bereits beschriebene diffuse Charakter des Sicherheitsbegriffes zu einer ebenso naheliegenden wie illegitimen Strategie. Die eigentliche inmitten der Garantiefrage liegende Gefahr besteht nämlich darin, dass auch ein Präventionsstaat im hier diskutierten Sinne - in dieser Facette einem Polizeistaat in der Tat nicht unähnlich - im Bestreben, eine Glaubwürdigkeitslücke zu vermeiden, der Versuchung unterliegt, eine ständige Drohkulisse unterhalten, die ihm argumentativ als Legitimation für die Unmöglichkeit der Abgabe von Garantien (und nebenbei gegebenenfalls als Grundlage für die stete Erhöhung der Eingriffsdichte und -tiefe sicherheitsbehördlicher Maßnahmen) dient. ${ }^{81}$ Auch hier gilt es insbesondere zu

76 Gusy, VVDStRL 63 (2004), 151, 170.

77 Gusy, VVDStRL 63 (2004), 151, 170 ff.; Beste, in: Lange (Hrsg.), Die Polizei der Gesellschaft, 2003, S. 285, 296 ff.; Pitschas, in: Pitschas/Stober, Kriminalprävention durch Sicherheitspartnerschaften, 2000, S. 4 f.; die dem Staat verbleibende Gesamtverantwortung gleicht damit einer Organisationsherrschaft oder, nach Möstl, Die staatliche Garantie für die öffentliche Sicherheit und Ordnung, 2002, S. 324, einer Garantenstellung. Zu Parallelen im Sozialstaatsprinzip vgl. BVerfGE 22, 180, 204.

78 Möstl, Die staatliche Garantie für die öffentliche Sicherheit und Ordnung, 2002, S. 318.

79 Der rechtliche Status des privaten Sicherheitsgewerbes, seine Befugnisse insbesondere im Verhältnis zu hoheitlichen Rechten und deren Trägern (und den Problemfeldern der Beleihung Privater, gemeinsamer Streifen mit der Polizei, police-private-partnerships, vgl. dazu Ochs, Die Polizei 2005, 7 ff.), seine Qualifikationen und seine möglichen Aktionsfelder (siehe dazu Ziercke, Die Polizei 2004, 331 ff.), die keinesfalls ausschlaggebend von der öffentlichen Haushaltslage bestimmt werden dürfen, sollten klar und über die Novellierung des Bewachungsgewerberechtes (BGBl. I 2002, 2724 ff.; vgl. dazu Schönleiter, GewArch 2003, 1 ff.), die DIN 77200 und das neue Berufsbild des »Geprüften Meisters für Schutz und Sicherheit « hinaus geregelt werden. Die derzeitigen Sicherheitspartnerschaften dürften diese Rahmenbedingungen eher verwischen als betonen; kritisch auch Waechter, JZ 2002, 854, 860. Erforderlich erscheint hier allerdings eher eine deutliche Abgrenzung zur hoheitlichen polizeilichen Tätigkeit denn ein z.B. von Pitschas unter anderem in: ders./Stolzlechner, Auf dem Weg in einen »neuen Rechtsstaat«, 2004, S. 20, postuliertes »Polizeikooperationsgesetz«. Zur staatlichen und privaten Sicherheitsgewährleistung insgesamt siehe Möstl, Die staatliche Garantie für die öffentliche Sicherheit und Ordnung, 2002, S. 290 ff.; Lenk/Prätorius (Hrsg.), Eingriffstaat und öffentliche Sicherheit, 1998.

80 Volkmann, JZ 2004, 696, 702.

81 So auch Düx, ZRP 2003, 189, 192; zur Frage der Legitimität damit einhergehender symbolischer Gesetzgebung Kötter, KJ 2003, 64, 69 f.. 
bedenken, dass die Zentrierung des Themas der inneren Sicherheit in hohem Maße der prinzipiellen Gefahr einer Anfälligkeit für medial noch beförderte Massenhysterien unterliegt. Als Remedium mit allerdings begrenzter Wirkung bietet sich in diesem Zusammenhang lediglich eine transparente und realistische Darstellung der Sicherheitslage an ${ }^{82}$ Letztlich bleibt damit nur ein möglicherweise naives Vertrauen auf die Redlichkeit der Verantwortlichen in Politik ${ }^{83}$ und Medien. ${ }^{84}$ Im Ergebnis gilt aber wiederum: wer der gesellschaftlichen Akzeptanz eines Perspektivwechsels von der sozialen zur inneren Sicherheit das Wort reden will, findet auch hier keine Gründe.

\section{Grenzen der Sicherheit}

Die Kehrseite der Frage nach der Garantie von Sicherheit ist die des Problems ihrer Grenzen, oder anders gewendet ihrer Maximierung. Es ist finalen Optimierungsaufgaben eigen, tendenziell nie vollkommen erfüllbar zu sein. ${ }^{85}$ Das gilt zunächst auch für soziale Wohltaten. Bei diesen lässt sich aber immerhin konstatieren, dass sie auf Grund ihrer materiellen Messbarkeit in ihrer Abhängigkeit vom Zustand des Staatsetats de facto nach oben hin tatsächlich begrenzt sind. ${ }^{86}$ Anderes gilt für die Grenzen des Präventionsstaates. Sicherheit als abstraktes Ziel im Sinne einer totalen Gefahrenabwehr und vollständigen Aufklärung aller Kriminalität - beides chimärenhafte polizeistaatliche Illusionen ${ }^{87}$ - lässt sich ins Unendliche steigern, denn nach der zunehmenden Ablösung des Begriffes der Gefahr durch den des Risikos ${ }^{88}$ wird sich, erst recht im Lichte der bereits erwähnten Volatilität und Irrationalität des Sicherheitsgefühls, für jede denkbare Situation ein zumindest in der Theorie prognostisch ad infinitum steigerbares Restrisiko bilden lassen. Die daraus erwachsende Gefahr ähnelt der oben beschriebenen: gerade weil die Skala der Sicherheit nach oben offen ist ${ }^{89}$ und eine Diskussion über den Umfang des zu tragenden Restrisikos nicht geführt wird, ${ }^{90}$ kann der Präventionsstaat seinen zu immer tiefgreifenderen Maßnahmen berechtigenden Sicherheitsanspruch theoretisch immer weiter und zu immer neuen Sicherheitsfeldern treiben.

Geht man im Versuch, der potentiellen Maßlosigkeit des Präventionsstaates einen Riegel vorzuschieben, der Frage nach den Grenzen legislativer und exekutivischer

82 In diesem Sinne auch Pitschas, JZ 1993, 857, 859 f.; Kötter, KJ 2003, 64, 71 f..

83 Siehe auch Paeffgen, GA 2003, 647, 662; Lange, Innere Sicherheit als Netzwerk, in: ders. (Hrsg.), Staat, Demokratie und Innere Sicherheit in Deutschland, 2000, S. 252.

84 Vgl. Pfeiffer/Windzio/Kleimann, MschrKrim 2004, 415 ff..

85 Schulze-Fielietz, in: FS Schmitt Glaeser, 2003, S. 407, 416.

86 Vgl. Volkmann, JZ 2004, 696, 700.

87 Vgl. Kaiser, Kriminologie, 3. Auflage 1996, § 17 Rn 1.

88 Gusy, VVDStRL 63 (2004), 151, 158 und 173 ff..

89 Schulze-Fielitz, in: FS Schmitt Glaeser, 2003, S. 407, 416; Ahlf, Die Polizei 2002, 93, 96; Denninger, in: Koch (Hrsg.), Terrorismus - Rechtsfragen der äußeren und inneren Sicherheit, 2002, S. 83, 89 f.; ders., KJ 2002, 467, 468 und 472; Gusy, VVDStRL 63 (2004), 151, 160 und 175.

90 Hoffmann-Riem, ZRP 2002, 497, 501; siehe aber Isensee, Grundrecht auf Sicherheit, 1983, S. $41 \mathrm{ff} .$. Letztlich handelt es sich hierbei um das zentrale Problem der gesamten Diskussion um den Präventionsstaat. 
Entwicklungen nach, so gelangt man wie oben bereits festgestellt zwangsläufig zunächst zum Grundsatz der Verhältnismäßigkeit als Lackmustest für Eingriffsbefugnisse. ${ }^{91}$ Darüber hinaus bedarf es weiterer gesetzlicher und von der Exekutive sorgfältig beachteter Kautelen. Darunter sind verfahrensrechtliche Absicherungen zu subsumieren wie Anordnungsvorbehalte für Eingriffsmaßnahmen, die Förderung der Transparenz staatlichen Handelns zumindest im Nachhinein sowie die Stärkung der Beschwerdemacht des Bürgers im Rechtswege und darüber hinaus. ${ }^{92}$

Hinzu tritt eine in viel höherem Maße als bisher praktizierte sachverständige legislative Beratung, Gesetzesfolgenabschätzung, Befristung und Evaluation. Vorschläge hierzu sind gerade in der jüngsten Vergangenheit zur Genüge gemacht worden. ${ }^{93} \mathrm{An}$ dererseits wird zu Recht darauf hingewiesen, dass symbolisches Recht gerade keiner Wirkungsforschung mehr bedarf, ja von dieser vielmehr als nur symbolisch entlarvt würde ${ }^{94}$ so dass sich die Motivation des einschlägigen Gesetzgebers hierzu damit in engen Grenzen halten dürfte. Bedauerlich ist auch, dass es derzeit noch an validen Kriterien und Verfahren für eine sicherheitsspezifische Evaluation mangelt. ${ }^{95}$

Schließlich ist besonderes Augenmerk auf Selbstverständnis und Handeln der Sicherheitsbehörden, namentlich der Polizei zu legen. Sie hat ihren Standort nicht auBerhalb der Gesellschaft, sondern ist selbst Bestandteil dieses komplexen, von verschiedensten Kräften und Gegenkräften bewegten und geprägten Systems. Als dessen Regulativ und Korrektiv eingerichtet, ist sie ihrerseits selbst dessen Veränderungen unterworfen. ${ }^{96}$ Professionelles polizeiliches Handeln bedarf somit in seiner präventiven wie auch seiner repressiven Ausformung einer steten und kritischen Analyse ers-

91 Dazu und zum Folgenden konkretisierend Schoch, Der Staat 43 (2004), 347, 367; SchulzeFielitz, in: FS Schmitt Glaeser, 2003, S. 407, 427 ff.; Trute, in: GS Jeand'Heur, 1999, S. 403, $413 \mathrm{ff}$. .

92 Die hierzu gemachten Vorschläge reichen von der Erweiterung der Funktion des Wehrbeauftragten auf die Sicherheitsbehörden (vgl. Pitschas, JZ 1993, 857, 865) bis hin zur Einrichtung von Ombudsmen (vgl. Gusy, VVDStRL 63 (2004), 151, 185).

93 Einen Überblick über die neuere Gesetzgebungslehre bieten z.B. Ennuschat, DVB1 2004, 986 ff.; Köck, VerwArch 2002, 1 ff.; aus Politikersicht siehe Thierse, NVwZ 2005, 153 ff.. Zur gemäß Art. 22 Abs. 3 des TerrorismusbekämpfungsG (BGBl I 2002, 361) vorzunehmenden Evaluation einzelner gemäß Art. 22 Abs. 2 des Gesetzes befristeter Befugnisse hat das Bundeskabinett am 11.05.2005 einen vom Bundesministerium des Innern vorgelegten Bericht beschlossen, der die Regelungen wenig überraschend (optimistisch noch Limbach, AnwBl 2002, 454, 457; dagegen grundsätzlich kritisch Frehsee, Der Rechtsstaat verschwindet, 2003, S. 294) als insgesamt erfolgreich und angemessen bezeichnet, eine weitere Befristung für entbehrlich hält und verschiedene Nachbesserungen fordert (vgl. die »Fakten zur Evaluierung des Terrorismusbekämpfungsgesetzes« unter www.bmi.bund.de). Der Umstand, dass die Evaluation offenbar vom Bundesministerium des Innern selbst vorgenommen worden ist, dürfte der Akzeptanz ihrer Ergebnisse allerdings abträglich sein. Zur Evaluation der Telefonüberwachung gemäß $\S \S 100 \mathrm{a}, \mathrm{b}$ StPO vgl. H.-J. Albrecht/ Dorsch/Krüpe, Rechtswirklichkeit und Effizienz der Überwachung der Telekommunikation, 2003; Backes/Gusy, Wer kontrolliert die Telefonüberwachung, 2003; siehe auch BMI (Hrsg.), Moderner Staat, Praxistest zur Gesetzesfolgenabschätzung, 2002.

94 Vgl. P.-A. Albrecht, Die vergessene Freiheit, 2003, S. 76; ders., Kriminologie, 2. Auflage 2002, S. 80; Denninger, ZRP 2002, 96, 101.

95 Gusy, VVDStRL 63 (2004), 151, 183. 
tens des Zustandes der Gesellschaft, in deren Auftrag sie tätig ist, und zweitens des Zustandes der Polizei selbst. Die vorstehend beschriebene Entwicklung fordert die Polizei als zunehmend letzte Legitimationsinstanz der Staatsgewalt in besonderem Maße heraus. Die an sie gestellten Anforderungen an ihre Glaubwürdigkeit werden steigen, sowohl hinsichtlich ihrer Rolle als streitschlichtende Bürgerpolizei als auch in ihrer Funktion als konsequente Verbrechensbekämpfer. Dabei muss Versuchen entgegengetreten werden, der Polizei die Aufgabe einer allgemeinen gesellschaftlichen Problemlösungsinstanz zuzuschreiben ${ }^{97}$ und sie damit zu überfordern. Das alles zwingt zur Überprüfung von Qualität und Quantität der personellen und materiellen polizeilichen Ressourcen. In personeller Hinsicht sind vor allem Rekrutierung, ${ }^{98}$ Aus- und Fortbildung und die Entwicklung eines adäquaten Berufsethos ${ }^{99}$ von Bedeutung, in materieller Hinsicht angemessene Ausstattung und Besoldung. Schließlich stellt sich in der Tat die Frage nach einer Generalrevision der nationalen und internationalen Sicherheitsapparaturen, deren Zukunft schon aus Gründen eines Machtgleichgewichtes und der gegenseitigen Kontrolle allerdings weniger in einer organisatorischen Zentralisierung als ein einer gelungenen Vernetzung liegen dürfte.

Auch hier sei abschließend die Frage nach dem Mehrwert gestellt. Die sorgfältige Beachtung aller dem Präventionsstaat immanenten Kautelen zum Zwecke seiner Begrenzung mag dem Bürger dessen Eingriffshandeln möglicherweise plausibel und damit erträglich zu machen. Daraus ergibt sich aber kein taugliches Argument dafür, der inneren Sicherheit eine Präferenz gegenüber anderen Staatszwecken zuzuweisen.

\section{Sicherheit als Werk der Gemeinschaft}

Der pädagogisch anspruchsvollste Inhalt der gegenwärtigen Reformen der Sozialsysteme dürfte den Maßnahmen zuzurechnen sein, die darauf abzielen, den Einzelnen aus seiner Empfängermentalität zu reißen und ihm ein höheres Maß an Initiative und Eigenverantwortung abzuverlangen. Ähnliches fordert bereits seit längerem die Kri-

96 Man denke abseits der Diskussion um den Präventionsstaat nur an demographische Faktoren. So wird die Alterung und Schrumpfung der Bevölkerung nicht nur die Kriminalität und deren Wahrnehmung verändern, sondern z.B. auch Einfluss nehmen auf die Rekrutierung der Polizei.

$97 \mathrm{Vgl}$. Behr, in: Organisationsentwicklung und die Probleme der Gestaltung von Veränderungsprozessen bei der Polizei, Schriftenreihe der Polizei-Führungsakademie, Band 2/2004, S. 143, 145, der die Polizei an der Schwelle vom Gerechtigkeitsparadigma zum Fürsorgeparadigma sieht; dazu schon Bull, in: ders. (Hrsg.), Sicherheit durch Gesetze?, 1987, S. 15, 17.

$98 \mathrm{Zu}$ den demographischen Aspekten polizeilicher Personalentwicklung vgl. Baldauf/Marx, in: Zielgerichtete Personalentwicklung bei den Polizeien des Bundes und der Länder, Schriftenreihe der Polizei-Führungsakademie, Band 3/2004, S. 14, 18 ff..

99 Die polizeilichen Ausbildungsstätten sind bundesweit seit Jahren um Professionalisierung und Akademisierung bemüht (vgl. Feltes/Punch, MSchrKrim 2005, 26, 36 ff.), wenngleich diese Bemühungen durch innerpolizeiliche Sozialisationsmuster konterkariert werden, innerhalb derer ein wohl weitgehend der in erster Linie kleinbürgerlichen Herkunft von Polizeibeamten einerseits und der sich erst in den letzten Jahren endgültig vom Militarismus verabschiedenden Ausbildung andererseits geschuldetes handwerklich-hemdsärmliges Berufsethos gepflegt wird, das sich einer Professionalisierung, die ihrem Inhalt nach zwangsläufig in eine Form von Akademisierung münden muss, hartnäckig widersetzt. 
minalprävention als integraler Bestandteil eines Präventionsstaates, der sich auf dem Gebiet der Gefahrenabwehr nicht ausschließlich hoheitlich gerieren will und kann. Prima vista findet sich in dieser Parallele eine dem Präventionsstaat eingebaute wirkungsvolle Sicherung gegen mögliche Exzesse: der Bürger ist, freilich nur in bestimmten, geeigneten Segmenten selbst zur Partizipation an der Aufrechterhaltung der öffentlichen Sicherheit aufgerufen, avanciert zum Protagonisten in einer Bürgergesellschaft und nimmt damit eine Rolle ein, die ihm ein Gegengewicht zu den Aktivitäten öffentlicher Handlungsträger mit Interventionskompetenz für die innere Sicherheit verleiht. Bei genauerer Betrachtung birgt dieser Anspruch allerdings Risiken. Gerade im Lichte der Erkentnisse der Kriminalprävention auf kommunaler Ebene, deren Erfolg nach allgemeiner Erfahrung zumeist davon abhängt, inwieweit die Polizei eine Lokomotivfunktion ausübt, ist schon äußerst fraglich, ob der Einzelne willens ist, eine derartige zusätzliche Verantwortung zu übernehmen. Die kriminalpolitische Erkenntnis, dass Kriminalprävention zuvörderst kommunale und gesamtgesellschaftliche Aufgabe sei, wurde von der Bevölkerung jedenfalls bislang ungeachtet einer durchaus anerkennenswerten Entwicklung ${ }^{100}$ nicht einmal ansatzweise flächendeckend internalisiert. ${ }^{101}$ Der Bürger begreift innere Sicherheit eben zunächst nicht als seine Sache, ${ }^{102}$ sondern als die des Staates. ${ }^{103}$ Bedenklich ist an diesem Ansinnen aber vor allem, dass der Präventionsstaat auf diese Weise in die Gefahr gerät, durch die teilweise Delegation ins Bürgerlich-Private seine eigene Autorität und Problemlösungskompetenz gerade in Frage zu stellen ${ }^{104}$ und damit ein schon mit dem Wachstum des privaten Sicherheitsgewerbes entstehendes Problem noch zu verschärfen. Unternimmt er nun andererseits, von der Fokussierung auf die innere Sicherheit als zunehmend letzte Bastion staatlicher Legitimation getrieben, die ihm eigentümlichen besonderen Anstrengungen insbesondere im Hinblick auf die Kumulation umfänglichen Informationswissens, ${ }^{105}$ gerät er letztlich in das Dilemma, widersprüchlich zu handeln.

100 Vgl. Fn. 52.

$101 \mathrm{Zu}$ den hierfür erforderlichen soziologischen Bedingungen siehe Gatzke, Die Polizei 2004, 349, 351; Kreissl, in: Stüllenberg (Hrsg.), Zukunftsstudie Organisation von Sicherheit in Deutschland 2013, 2003, S. 35, 54 ff..

102 So eine verschiedentlich geäußerte Idealvorstellung, z.B. von Kötter, Der Staat 43 (2004), 371, 383 ff.; Kniesel, ZRP 1996, 482, 489.

103 Selbst ein ausgewiesener Apologet der Privatisierung wie Pitschas konstatiert, dass das derzeitige zivilgesellschaftliche Netzwerk zur Garantie der inneren Sicherheit eher schlecht als recht funktioniere, vgl. Pitschas, in: ders./Stolzlechner (Hrsg.), Auf dem Weg in einen »neuen Rechtsstaat«, 2004, S. 17; Gusy, VVDStRL 63 (2004), 151, 162; Kreissl, in: Stüllenberg (Hrsg.), Zukunftsstudie Organisation von Sicherheit in Deutschland 2013, 2003, S. 35, 62 ff.; Frehsee, Der Rechtsstaat verschwindet, 2003, S. 325 f..

104 Dem Argument, der Staat konzentriere sich eben auf die wesentlichen Bedrohungen der inneren Sicherheit und überlasse den Rest des Feldes dem Bürger, ließe sich die provokative Frage entgegenhalten, wie dem Staat denn die Aufrechterhaltung der inneren Sicherheit gelingen solle, wenn er schon im Kleinen den Rückzug antritt; siehe auch Beste, in: Lange (Hrsg.), Die Polizei der Gesellschaft, 2003, S. 285, 297 f..

105 Kritisch Denninger, KJ 2002, 467, 473; relativierend allerdings ders., KritV 2003, 313, 320 f.. Zum Postulat einer umfassenden Informationsvorsorge auch im Rahmen des Sozialstaatsprinzips siehe Aulehner, Polizeiliche Gefahren- und Informationsvorsorge, 1998, S. 440 ff. 
Damit aber nicht genug: er tritt zugleich dem von diesen Rechtseingriffen betroffenen Bürger problematischerweise nochmals zu nahe. ${ }^{106}$ Dieser hat nämlich gleichzeitig zweierlei zu gewärtigen: erstens sich mit der Aufforderung konfrontiert zu sehen, sich über bloßes rechtstreues Verhalten hinaus selbst präventiv zu engagieren, und dessen ungeachtet zweitens auch als Nichtverdächtiger in zunehmendem Maße mit hoheitlichen Maßnahmen überzogen zu werden. Ob dem ohnehin bereits von sozialstaatlichen Forderungen nach höherer Eigenverantwortung betroffenen Einzelnen diese weiteren Inanspruchnahmen vermittelt werden können, darf füglich bezweifelt werden.

Ein Weiteres tritt hinzu: Der Aufruf zu bürgergesellschaftlichem Engagement in sozialen Bereichen ist dem Grunde nach Ausdruck eines solidarisch-philanthropischen Menschenbildes, dem Eingriffe in Rechte anderer wesensfremd sind. Bürgerliche Selbsthilfe in Sachen innerer Sicherheit hingegen funktioniert dem Grunde nach nicht nach dem Prinzip des Vertrauens, sondern des Misstrauens gegenüber dem Mitmenschen. Zudem bewegt es sich an der Grenze informeller zu formeller Sozialkontrolle und damit tendenziell im Bereich von Eingriffen in Rechte anderer. Ohne privatem

106 Zum Mechanismus, der den Bürger auf Grund eines vom Staat zu gewährleistenden »Grundrechtes « auf Sicherheit und Schutz vom Grundrechtssubjekt bestehender Freiheitsverbürgungen zum Grundpflichtadressaten avancieren lässt, siehe z.B. Denninger, KJ 1988, 1, 13. Einen anderen Ansatz wählen Buckel/Kannankulam, Das Argument 44 (2002), 34 ff., die gerade in der Verlegung von Sicherheitsaktivitäten in die Selbstorganisationsfähigkeit des Einzelnen den Grund dafür sehen, dass Elemente der Überwachung ebenfalls auf diese Ebene verlagert werden und diese durchdringen müssen. Die Autarkisierung des Bürgers zwänge so zu dessen verstärkter Überwachung. Kötter, Der Staat 43 (2004) 371, 383 f. hingegen vermag an einer Teilaufgabe von Privatsphäre zum Zwecke der Gewährleistung kognitiver Sicherheit nichts auszusetzen. In seinem der Arbeitspsychologie entlehnten Ansatz der »kontrollierten Autonomie « erfahren Maßnahmen wie beispielsweise die öffentliche Videoüberwachung ihre Rechtfertigung erstens in ihrer Erinnerungsfunktion für den Bürger, seinem freiwilligen Selbstzwang zu normgerechtem Verhalten auch tatsächlich nachzukommen, und zweitens in dem Umstand, dass der Staat alle Bürger gleichermaßen mit solchen Eingriffen überzieht. Dabei geht Kötter allerdings von der optimistischen Annahme aus, dass der Bürger im Wissen um die Notwendigkeit der Überwachung sich dieser mit vollem Einverständnis stellt, sie bewusst als Medium der Selbstkontrolle nutzt und damit gleichsam für sich vereinnahmt. Diese der Überwachung letztlich ihren Eingriffscharakter nehmende Vermutung darf schon in ihrer Prämisse - hier bleibt unklar, ob sie ein grundsätzliches Wissen des Bürgers über Art und Umfang der gegen ihn gerichteten Überwachungsmaßnahmen voraussetzt - als auch in ihren Folgen für das Grundrechtsverständnis bezweifelt werden; die im Volkszählungsurteil in BVerfGE 65, 1, 43 ausgesprochenen Befürchtungen, eine Person, die nicht die Herrschaft über ihre Daten besitze, verzichte deshalb unter Umständen auf die Ausübung von Grundrechten, erscheinen bei Kötter jedenfalls in ganz neuem Licht. In Kötters Richtung zielt offenbar auch Quambusch, Kriminalistik 2005, 156, 159, der der bundesdeutschen Gesellschaft eine geschichts- und religionsbedingte Sozialisation attestiert, die von einer gewissen Affinität gegenüber staatlichen Überwachungsmaßnahmen gekennzeichnet sein soll. Realistischer, gleichzeitig aber auch bedrohlicher scheint die Beobachtung zu sein, dass dem Bürger eine umfassende Überwachung tatsächlich gleichgültig ist, wie es Herzinger, Die Zeit 32/2003, S. 1 vermutet, der von der Gefahr eines »Bequemlichkeits-Totalitarismus « spricht. Siehe im Übrigen auch Prömmel, KrimJ 2002, 242, 256; den Sanktionsgedanken betonend Hesse, Der Schutzstaat, 1994, S. 116; von einem weiten Präventionsbegriff ausgehend Grimm, Die Zukunft der Verfassung, 1991, S. 418. 
Engagement zum Erhalt der inneren Sicherheit die Existenzberechtigung absprechen zu wollen: als Paradigma eines gesellschaftlichen Selbstverständnisses wirkt es eher bedrohlich denn beruhigend. ${ }^{107}$

\section{Ergebnis}

Nimmt man dem Gedanken des Präventionsstaats durch die Verpflichtung auf rechtsstaatliche Regeln seinen Absolutheitsanspruch, verliert der Begriff zunächst viel von seinem Stigma. Gleichzeitig erinnert er an eine Verschmelzung des Konzepts klassischer staatlicher Sicherheitsapparate mit der im Rahmen eines technikzentrierten Sicherheitsverständnisses entwickelten Idee eines Versicherungsstaates als gesellschaftliche Architektur, die nach dem Modell der kollektiven Assekuranz gegen Risiken funktioniert, also auf der Vergesellschaftung von Gefahr und Verantwortung. ${ }^{108}$ Dass sich eine dazu erforderliche von der gesamten Bevölkerung getragene Bereitschaft zur Beitragzahlung dauerhaft einfordern lässt, bedarf allerdings noch des Nachweises. Vor allem aber erweist sich der konkrete Ertrag legislativer und exekutiver Schwerpunktsetzungen der inneren Sicherheit als viel zu vage und medial manipulierbar, als dass sich darauf ein tragfähiges dominantes Staatsverständnis gründen ließe. Ohne notwendigerweise in die Kassandra-Rufe vorzugsweise liberaler Autoren einstimmen zu müssen, die vor einem allzu forsch eingeschlagenen Weg in den Präventionsstaat warnen, so ist jedenfalls zu bezweifeln, ob es ratsam ist, den sozialen Staat in der Annahme, innerer Friede gelänge ohne sozialen Frieden, in einer sich immer weiter ausdifferenzierenden Gesellschaft paradigmatisch durch den Präventionsstaat zu ersetzen. ${ }^{109}$ Soziale und innere Sicherheit sind Themenfelder von zu zentraler Bedeutung für die Befriedigung gesellschaftlicher Bedürfnisse, als dass sie sich gegenseitig aufrechnen ließen. ${ }^{110}$

Abgesehen davon: es würde zwar den Grundsätzen der Kybernetik widersprechen, einem komplexen System mit einfachen Regelungsmechanismen begegnen zu wollen. Dennoch könnte es in der Diskussion um den Präventionsstaat hilfreich sein, sich frei von Polemik auf eine alte, ebenso banale wie zutreffende, gleichzeitig aber auch ernüchternde polizeitaktische Weisheit zu besinnen: wer alles schützen will, schützt nichts.

107 Um mit den Worten Hoffmann-Riems, ZRP 2002, 497, 501 zu sprechen: eine Reduzierung der Gesellschaft auf innere Sicherheit droht das Lebensgefühl zu lähmen und Empathie für andere und Toleranz für Andersartige zu unterdrücken.

108 Vgl. Beck, Gegengifte, 1988, S. 179 ff. unter Berufung auf Ewald, L'Etat providence, 1986 (Der Vorsorgestaat, 1993), und gleichzeitiger Kritik an dessen Ansatz. Dieser ist übrigens nicht deckungsgleich mit einem Staatsverständnis als »Versicherungsverein auf Gegenseitigkeit« im Sinne von Isensee, Grundrecht auf Sicherheit, 1983, S. 13, sondern reicht darüber hinaus.

109 Gegenmodellen wie dem des globalen Sozialstaates, wie er von Düx, ZRP 2003, 189, 195 gefordert wird, der damit im Grunde genommen den alten Satz v. Liszts (vgl. Fn 61) neu und mondial interpretiert (in diese Richtung auch Hassemer, Vorgänge 3/2002, 10, 15) fehlt es freilich erst recht an Konkurrenzfähigkeit.

110 So auch Ress, VVDStRL 48 (1990), 56, 111, der eine Rangfolge ablehnt. 